\title{
Involutive triangular matrix algebras
}

\author{
Morteza Ahmadi (D), Ahmad Moussavi* (D) \\ Department of Pure Mathematics, Faculty of Mathematical Sciences, Tarbiat Modares University, \\ P.O.Box:14115-134, Tehran, Iran
}

\begin{abstract}
In this paper we provide new examples of Banach $*$-subalgebras of the matrix algebra $\mathbf{M}_{n}(\mathscr{A})$ over a commutative unital $C^{*}$-algebra $\mathscr{A}$. For any involutive algebra, we define two involutions on the triangular matrix extensions. We prove that the triangular matrix algebras over any commutative unital $C^{*}$-algebra are Banach $*$-algebras and that the primitive ideals of these algebras and some of their Banach $*$-subalgebras are all maximal.
\end{abstract}

Mathematics Subject Classification (2010). 15A60, 16D60, 46H10, 46K05

Keywords. primitive ideal, maximal ideal, Banach *-algebra, $\mathrm{C}^{*}$-algebra

Involutive rings and linear algebras have been investigated after von Neumanns recognition of the importance of the canonical adjoint of the algebra of bounded operators on spaces in the study of what are now called von Neumann algebras. For instance, I.N. Herstein and Susan Montgomery studied the algebraic structures of rings with involutions resulting in nice results. Also, such rings have been investigated in the context of applications to Lie algebras, Jordan algebras, Banach $*$-algebras, and von Neumann algebras.

Let $n$ be an integer with $n \geq 2$. For any unital algebra $\mathscr{A}$, let $\mathbf{M}_{n}(\mathscr{A})$ denote the algebra of $n \times n$ matrices over $\mathscr{A}$ and let $\mathbf{T}_{n}(\mathscr{A})$ denote the algebra of $n \times n$ upper triangular matrices over $\mathscr{A}$. Let $V_{n}=\sum_{i=1}^{n-1} E_{i, i+1}$, where $E_{i j}, 1 \leq i, j \leq n$, is the matrix with 1 in the $(i, j)$-position and 0 elsewhere. Following Lee and Zhou [4], we define

$$
\mathbf{A}_{n}(\mathscr{A})=\mathscr{A} I_{n}+\sum_{\ell=2}^{\left[\frac{n}{2}\right]} \mathscr{A} V_{n}^{\ell-1}+\sum_{i=1}^{\left[\frac{n+1}{2}\right]} \sum_{j=\left[\frac{n}{2}\right]+i}^{n} \mathscr{A} E_{i, j},
$$

and

$$
\mathbf{B}_{n}(\mathscr{A})=\mathscr{A} I_{n}+\sum_{\ell=3}^{\left[\frac{n}{2}\right]} \mathscr{A} V_{n}^{\ell-2}+\sum_{i=1}^{\left[\frac{n+1}{2}\right]+1} \sum_{j=\left[\frac{n}{2}\right]+i-1}^{n} \mathscr{A} E_{i, j},
$$

where $[x]$ is called the integer part of $x, x \in \mathbb{R}$.

\footnotetext{
*Corresponding Author.

Email addresses: morteza.ahmadi23@gmail.com (M. Ahmadi), moussavi.a@gmail.com (A. Moussavi)

Received: 02.05.2019; Accepted: 22.01.2020
} 
The algebra $\mathbf{S}_{n}(\mathscr{A})$ is defined as a subalgebra of $\mathbf{T}_{n}(\mathscr{A})$ by

$$
\mathbf{S}_{n}(\mathscr{A})=\mathscr{A} I_{n}+\sum_{i<j} \mathscr{A} E_{i, j}
$$

Also, the algebra $\mathbf{V}_{n}(\mathscr{A})$ is defined as a subalgebra of $\mathbf{S}_{n}(\mathscr{A})$ by

$$
\mathbf{V}_{n}(\mathscr{A})=\mathscr{A} I_{n}+\sum_{\ell=2}^{n} \mathscr{A} V_{n}^{\ell-1}
$$

Furthermore, we introduce

$$
\mathbf{U}_{n}(\mathscr{A})=\mathscr{A} I_{n}+\sum_{i=1}^{\left[\frac{n-1}{2}\right]} \sum_{j=\left[\frac{n}{2}\right]+1}^{n} \mathscr{A} E_{i, j}+\sum_{j=\left[\frac{n-1}{2}\right]+2}^{n} \mathscr{A} E_{\left[\frac{n-1}{2}\right]+1, j} .
$$

We define two involutions $*$ and $\star$ on the triangular matrix algebra over any unital $*$-algebra. Suppose that $\mathscr{A}$ is a unital algebra with an involution $*$, and for each $n \geq 2$ consider the algebra $\mathbf{M}_{n}(\mathscr{A})$. Let $*$ denote the $*$-transpose involution on $\mathbf{M}_{n}(\mathscr{A})$. Put $E=\sum_{i+j=n+1} E_{i j}$. We define an involution $*$ on $\mathbf{M}_{n}(\mathscr{A})$ by $A^{*}=E A^{*} E$, for $A=$ $\left(a_{i j}\right) \in \mathbf{M}_{n}(\mathscr{A})$. It is easy to check that $A^{*}=\left(a_{\ell k}^{*}\right)$, where $\ell=n-j+1$ and $k=n-i+1$.

We show that the subalgebra $\mathbf{U}_{n}(\mathscr{A})$ is closed under the involution $*$. We prove the case when $n=2 m$ is an even number. The case of odd numbers can be shown similarly. Let $A=a I_{2 m}+\sum_{i=1}^{m-1} \sum_{j=m+1}^{2 m} a_{i, j} E_{i, j}+\sum_{j=m+1}^{2 m} a_{m, j} E_{m, j} \in \mathbf{U}_{2 m}(\mathscr{A})$. Then

$$
\begin{aligned}
A^{*}=a^{*} I_{2 m} & +\sum_{i=1}^{m-1} \sum_{j=m+1}^{2 m} a_{2 m-j+1,2 m-i+1}^{*} E_{2 m-j+1,2 m-i+1} \\
& +\sum_{j=m+1}^{2 m} a_{2 m-j+1, m+1}^{*} E_{2 m-j+1, m+1} .
\end{aligned}
$$

Set $i^{\prime}=2 m-j+1$ and $j^{\prime}=2 m-i+1$. Then

$$
\begin{aligned}
A^{*} & =a^{*} I_{2 m}+\sum_{i^{\prime}=1}^{m} \sum_{j^{\prime}=m+2}^{2 m} a_{i^{\prime}, j^{\prime}}^{*} E_{i^{\prime}, j^{\prime}}+\sum_{i^{\prime}=1}^{m} a_{i^{\prime}, m+1}^{*} E_{i^{\prime}, m+1} \\
& =a^{*} I_{2 m}+\sum_{i^{\prime}=1}^{m-1} \sum_{j^{\prime}=m+2}^{2 m} a_{i^{\prime}, j^{\prime}}^{*} E_{i^{\prime}, j^{\prime}}+\sum_{j^{\prime}=m+2}^{2 m} a_{m, j^{\prime}}^{*} E_{m, j^{\prime}}+\sum_{i^{\prime}=1}^{m} a_{i^{\prime}, m+1}^{*} E_{i^{\prime}, m+1} \\
& =a^{*} I_{2 m}+\sum_{i^{\prime}=1}^{m-1} \sum_{j^{\prime}=m+2}^{2 m} a_{i^{\prime}, j^{\prime}}^{*} E_{i^{\prime}, j^{\prime}}+\sum_{j^{\prime}=m+2}^{2 m} a_{m, j^{\prime}}^{*} E_{m, j^{\prime}}+\sum_{i^{\prime}=1}^{m-1} a_{i^{\prime}, m+1}^{*} E_{i^{\prime}, m+1} \\
& +a_{m, m+1}^{*} E_{m, m+1} \\
& =a^{*} I_{2 m}+\sum_{i^{\prime}=1}^{m-1} \sum_{j^{\prime}=m+1}^{2 m} a_{i^{\prime}, j^{\prime}}^{*} E_{i^{\prime}, j^{\prime}}+\sum_{j^{\prime}=m+2}^{2 m} a_{m, j^{\prime}}^{*} E_{m, j^{\prime}} \in \mathbf{U}_{2 m}(\mathscr{A}) .
\end{aligned}
$$

By inspection, we see that the subalgebras $\mathbf{T}_{n}(\mathscr{A}), \mathbf{S}_{n}(\mathscr{A}), \mathbf{A}_{n}(\mathscr{A}), \mathbf{B}_{n}(\mathscr{A})$, and $\mathbf{V}_{n}(\mathscr{A})$ of $\mathbf{M}_{n}(\mathscr{A})$ are also self-adjoint with respect to the involution $*$. Thus these are also *-algebras.

Now, we define the second involution. Suppose that $\mathscr{A}$ is a unital algebra with an involution $*$, and for each $n=2 m$ with $m \in \mathbb{N}$ consider the following matrix:

$$
D=\sum_{i=1}^{m} E_{i i}-\sum_{i=m+1}^{n} E_{i i}=\left(\begin{array}{cc}
\mathrm{I}_{m} & 0 \\
0 & -\mathrm{I}_{m}
\end{array}\right) \in \mathbf{M}_{n}(\mathscr{A}) .
$$


We define an involution $\star$ on $\mathbf{M}_{n}(\mathscr{A})$ by $A^{\star}=D A^{*} D$, for each $A \in \mathbf{T}_{n}(\mathscr{A})$. It is easy to see that $\mathbf{T}_{n}(\mathscr{A}), \mathbf{S}_{n}(\mathscr{A})$, and $\mathbf{U}_{n}(\mathscr{A})$ are self-adjoint with respect to the involution $\star$. Thus these are also $*$-algebras.

Definition 1 ([5]). Let $\mathscr{A}$ be an algebra over $\mathbb{C}$. Then $\mathscr{A}$ is called a normed algebra if there is a norm $\|\cdot\|$ on $\mathscr{A}$ satisfying the following condition:

(i) $\|x y\| \leq\|x\|\|y\|$, for all $x, y \in \mathscr{A}$.

A normed algebra $\mathscr{A}$ is called a Banach algebra if it is complete in the norm. If, in addition, $\mathscr{A}$ has a unit 1 such that $\|1\|=1$, it is called a unital Banach algebra. If a normed algebra $\mathscr{A}$ admits an involution $*$ with the following properties:

(ii) $(\alpha x)^{*}=\bar{\alpha} x^{*}$,

(iii) $\left\|x^{*}\right\|=\|x\|$,

for every $x \in \mathscr{A}$ and every $\alpha \in \mathbb{C}$, then $\mathscr{A}$ is called a normed $*$-algebra. A Banach *-algebra (or involutive normed algebra) is a complete normed *-algebra.

A $C^{*}$-norm on a complex *-algebra $\mathscr{A}$ is a norm $\|\cdot\|$ satisfying Condition (i) and

(iv) $\left\|x^{*} x\right\|=\|x\|^{2}$, for all $x \in \mathscr{A}$.

A complex $*$-algebra equipped with a $\mathrm{C}^{*}$-norm is called a pre- $C^{*}$-algebra. If $\mathscr{A}$ is a pre$\mathrm{C}^{*}$-algebra which is complete in the norm then it is called a $C^{*}$-algebra.

Remark 2. The matrix algebra $\mathbf{M}_{n}(\mathbb{C})$ is a $\mathrm{C}^{*}$-algebra with the conjugate transpose involution $*$ and the operator norm defined by

$$
\|T\|=\sup \left\{\frac{\|T X\|}{\|X\|} \mid 0 \neq X \in \mathbb{C}^{n}\right\}, T \in \mathbf{M}_{n}(\mathbb{C}) .
$$

Note that the norm of a column matrix $X$ in the above definition is the usual Euclidean norm induced by the standard inner product of $\mathbb{C}^{n}$. It is a standard fact from linear algebra that $\|T\|=\sqrt{\lambda_{\max }\left(T T^{*}\right)}$, where $T \in \mathbf{M}_{n}(\mathbb{C}), *$ is the transpose involution of $\mathbf{M}_{n}(\mathbb{C})$, and $\lambda_{\max }\left(T T^{*}\right)$ is the greatest eigenvalue of $T T^{*}$.

Lemma 3. The normed *-algebra $\boldsymbol{T}_{n}(\mathbb{C})$ with involution $*$ is a Banach $*$-algebra.

Proof. Equip $\mathbf{T}_{n}(\mathbb{C})$ with the operator norm of $\mathbf{M}_{n}(\mathbb{C})$. Since $\mathbf{M}_{n}(\mathbb{C})$ with the operator norm is a Banach algebra (and hence so is $\mathbf{T}_{n}(\mathbb{C})$ ), to show that $\mathbf{T}_{n}(\mathbb{C})$ is a Banach $*$ algebra it is enough to prove the condition (iii) in Definition 1, that is, $\|A\|=\left\|A^{*}\right\|$, for each $A \in \mathbf{T}_{n}(\mathbb{C})$. First note that, it suffices to show that $\|A\|=\left\|A^{*}\right\|$ for each invertible matrix $A \in \mathbf{T}_{n}(\mathbb{C})$. To see this, let $A \in \mathbf{T}_{n}(\mathbb{C})$ and set $A_{n}=A+\frac{1}{n} I_{n}, n \in \mathbb{N}$. Thus $A_{n}^{*}=A^{*}+\frac{1}{n} I_{n}$. Since $A$ has only finitely many eigenvalues, there exists an integer $N$ such that for each $n \geq N, A_{n}$ is invertible. Now $A_{n} \rightarrow A$ and $A_{n}^{*} \rightarrow A^{*}$ in the operator norm and so $\left\|A_{n}\right\| \rightarrow\|A\|$ and $\left\|A_{n}^{*}\right\| \rightarrow\left\|A^{*}\right\|$. Therefore, the equalities $\left\|A_{n}\right\|=\left\|A_{n}^{*}\right\|$, $n \geq N$, imply that $\|A\|=\left\|A^{*}\right\|$.

Now suppose that $A=\left(a_{i j}\right) \in \mathbf{T}_{n}(\mathbb{C})$ is an invertible upper triangular matrix. Then $a_{i i} \neq 0$, for $1 \leq i \leq n, A^{*}=\left(\overline{a_{j i}}\right), A^{*}=\left(\overline{a_{n-j+1, n-i+1}}\right)$, and $\left(A^{*}\right)^{*}=\left(a_{n-i+1, n-j+1}\right)$. Let $C=A A^{*}=\left(c_{i, j}\right)$ and $D=A^{*}\left(A^{*}\right)^{*}=\left(d_{i, j}\right)$. Thus,

$$
c_{i, j}=\sum_{k=1}^{n} a_{i, k} \overline{a_{j, k}}, \quad d_{i, j}=\sum_{k=1}^{n} \overline{a_{n-k+1, n-i+1}} a_{n-k+1, n-j+1} .
$$


We shall show that $\lambda_{\max }(C)=\lambda_{\max }(D)$. For this, we show that $C$ and $D$ are similar matrices. Set

$$
P=\left(\begin{array}{ccccc}
a_{1, n} & a_{1, n-1} & \cdots & a_{1,2} & a_{1,1} \\
a_{2, n} & a_{2, n-1} & \cdots & a_{2,2} & 0 \\
\vdots & \vdots & . & \vdots & \vdots \\
a_{n-1, n} & a_{n-1, n-1} & \cdots & 0 & 0 \\
a_{n, n} & 0 & \cdots & 0 & 0
\end{array}\right),
$$

and let $P D=\left(x_{i, j}\right)$ and $C P=\left(y_{i, j}\right)$. Then

$$
\begin{aligned}
x_{i, j} & =\sum_{k=1}^{n} \sum_{\ell=1}^{n} a_{i, n-k+1} \overline{a_{n-\ell+1, n-k+1}} a_{n-\ell+1, n-j+1}, \\
y_{i, j} & =\sum_{k=1}^{n} \sum_{\ell=1}^{n} a_{k, n-j+1} \overline{a_{k, \ell}} a_{i, \ell} .
\end{aligned}
$$

Now we have $P D=C P$. In fact, write $k^{\prime}=n-\ell+1$ and $\ell^{\prime}=n-k+1$. Then we have

$$
x_{i, j}=\sum_{k=1}^{n} \sum_{\ell=1}^{n} a_{i, n-k+1} \overline{a_{n-\ell+1, n-k+1}} a_{n-\ell+1, n-j+1}=\sum_{k^{\prime}=1}^{n} \sum_{\ell^{\prime}=1}^{n} a_{k^{\prime}, n-j+1} \overline{a_{k^{\prime}, \ell^{\prime}}} a_{i, \ell^{\prime}}=y_{i, j} .
$$

The matrix $P$ is invertible since we have

$$
\begin{aligned}
\operatorname{det}(P) & =(-1)^{n+1} a_{1,1}(-1)^{n} a_{2,2} \cdots(-1)^{3} a_{n-1, n-1}(-1)^{2} a_{n, n} \\
& =a_{1,1} a_{2,2} \cdots a_{n, n}(-1)^{\frac{n(n+3)}{2}} \\
& =(-1)^{\frac{n(n+3)}{2}} \operatorname{det}(\mathscr{A}) .
\end{aligned}
$$

Hence $D=P^{-1} C P$ which implies that $D$ and $C$ have the same eigenvalues. Therefore, $\|A\|=\sqrt{\lambda_{\max }(C)}=\sqrt{\lambda_{\max }(D)}=\left\|A^{*}\right\|$.

We want to replace $\mathbb{C}$ in Lemma 3 with a commutative $\mathrm{C}^{*}$-algebra. This gives a source of interesting examples. For this we need some preparation. Let $\mathscr{A}$ be a $\mathrm{C}^{*}$-algebra and let $n \geq 2$. Consider the $*$-algebra $\mathbf{T}_{n}(\mathscr{A})$ of upper triangular matrices with entries in $\mathscr{A}$ and the involution $*$. Equip $\mathbf{T}_{n}(\mathscr{A})$ with the norm of the $\mathrm{C}^{*}$-algebra $\mathbf{M}_{n}(\mathscr{A})$ (see [5, Section 3.4] and [7, Chapter 1] for the definition of this norm).

Theorem 4. Let $\mathscr{A}$ be a commutative $C^{*}$-algebra and let $\boldsymbol{T}_{n}(\mathscr{A})$ be the normed $*$-algebra defined above. Then $\boldsymbol{T}_{n}(\mathscr{A})$ is a Banach *-algebra.

Proof. Let $\mathscr{A}$ be a commutative $\mathrm{C}^{*}$-algebra. Then there is a locally compact Hausdorff space $X$ such that $\mathscr{A} \cong \mathrm{C}_{0}(X)[5]$. Thus we may assume that $\mathscr{A}=\mathrm{C}_{0}(X)$. Let $S=\left(a_{i j}\right) \in$ $\mathbf{T}_{n}\left(\mathrm{C}_{0}(X)\right)$. The map $\varphi: \mathbf{M}_{n}\left(\mathrm{C}_{0}(X)\right) \rightarrow \mathrm{C}_{0}\left(X, \mathbf{M}_{n}(\mathbb{C})\right)$ defined by $\varphi\left(\left(f_{i j}\right)\right)(x)=\left(f_{i j}(x)\right)$, for $\left(f_{i j}\right) \in \mathbf{M}_{n}\left(\mathrm{C}_{0}(X)\right)$ and $x \in X$, is a $*$-isomorphism of $\mathrm{C}^{*}$-algebras (see e.g., [7]). For each $x \in X$ set $S(x)=\varphi(S)(x)=\left(a_{i j}(x)\right) \in \mathbf{M}_{n}(\mathbb{C})$. By Lemma $3,\left\|S(x)^{*}\right\|=\|S(x)\|$, $x \in X$. Thus we have

$$
\left\|S^{*}\right\|=\sup _{x \in X}\left\|S(x)^{*}\right\|=\sup _{x \in X}\|S(x)\|=\|S\| .
$$

Therefore, $\mathbf{T}_{n}(\mathscr{A})$ is a Banach $*$-algebra.

Corollary 5. Let $\mathscr{A}$ be a commutative $C^{*}$-algebra and let $n \geq 2$. Then the $*$-algebras $\boldsymbol{S}_{n}(\mathscr{A}), \boldsymbol{A}_{n}(\mathscr{A}), \boldsymbol{B}_{n}(\mathscr{A}), \boldsymbol{U}_{n}(\mathscr{A})$, and $\boldsymbol{V}_{n}(\mathscr{A})$ with involution $*$ are Banach $*$-subalgebras of $\boldsymbol{T}_{n}(\mathscr{A})$.

Now we deal with the other involution $\star$. As in Theorem 4 , we equip $\mathbf{T}_{n}(\mathscr{A})$ with the norm of the $\mathrm{C}^{*}$-algebra $\mathbf{M}_{n}(\mathscr{A})$. 
Theorem 6. Let $\mathscr{A}$ be a commutative $C^{*}$-algebra and let $n \geq 2$ be an even integer. Then the normed $*$-algebra $\boldsymbol{T}_{n}(\mathscr{A})$ with involution $\star$ is a Banach $*$-algebra.

Proof. As in the proof of Lemma 3, it is enough to verify Condition (iii) in Definition 1 for $\mathbf{T}_{n}(\mathscr{A})$, that is, $\|S\|=\left\|S^{\star}\right\|$, for each $S \in \mathbf{T}_{n}(\mathscr{A})$. Let $S \in \mathbf{T}_{n}(\mathscr{A})$. We consider the matrix $D$ in the unital algebra $\mathrm{M}_{n}\left(\mathscr{A}^{\sim}\right)$, where $\mathscr{A}^{\sim}$ denotes the unitization of $\mathscr{A}$, i.e., $\mathscr{A}^{\sim}=\mathscr{A} \oplus \mathbb{C}$ (see [5, Section 1.2]), since $\mathscr{A}$ may not be unital. Note that $D=D^{-1}=D^{*}$ and hence $D$ is a unitary in $\mathrm{M}_{n}\left(\mathscr{A}^{\sim}\right)$. Thus $\|D\|=1$. Then we have

$$
\left\|S^{\star}\right\|=\left\|D S^{*} D\right\| \leq\|D\| \cdot\left\|S^{*}\right\| \cdot\|D\|=\left\|S^{*}\right\| .
$$

Thus $\left\|S^{\star}\right\| \leq\left\|S^{*}\right\|$. On the other hand, by Theorem $4,\left\|S^{*}\right\|=\|S\|$. Hence, $\left\|S^{\star}\right\| \leq\|S\|$. By replacing $S$ with $S^{\star}$ we get $\|S\|=\left\|\left(S^{\star}\right)^{\star}\right\| \leq\left\|S^{\star}\right\|$. Therefore, $\left\|S^{\star}\right\|=\|S\|$, and so $\mathbf{T}_{n}(\mathbb{C})$ is a Banach $*$-algebra.

Corollary 7. Let $\mathscr{A}$ be a commutative $C^{*}$-algebra and let $n \geq 2$ be an even integer. Then the $*$-algebras $\boldsymbol{S}_{n}(\mathscr{A})$ and $\boldsymbol{U}_{n}(\mathscr{A})$ with involution $\star$ are Banach $*$-subalgebras of $\boldsymbol{T}_{n}(\mathscr{A})$.

The following result (i.e., the existence of finite dimensional complex $*$-algebras that do not admit any $\mathrm{C}^{*}$-norm, but at the same time they have Banach $*$-algebra structures) is not well known in the theory of operator algebras.

Remark 8. Let $\mathscr{A}$ be a commutative unital $\mathrm{C}^{*}$-algebra and $n \geq 2$. Then there is no $\mathrm{C}^{*}$-norm on the finite dimensional complex $*$-algebras $\mathbf{T}_{n}(\mathscr{A})$.

Proof. Assume on the contrary that there is a $\mathrm{C}^{*}$-norm on $\mathbf{T}_{n}(\mathscr{A})$, say \|\| . Then

$$
\left\|E_{1, n}\right\|^{2}=\left\|E_{1, n} E_{1, n}^{*}\right\|=0 .
$$

So $\left\|E_{1, n}\right\|=0$, which is a contradiction. Thus there is no $\mathrm{C}^{*}$-norm on $\mathbf{T}_{n}(\mathscr{A})$.

Let $\mathscr{A}$ be an algebra with unity and $\mathcal{H}$ a Hilbert space. Recall from [2] that, a representation of $\mathscr{A}$ in $\mathcal{H}$ is an algebra homomorphism $\pi$ from $\mathscr{A}$ into the algebra $\mathscr{L}(\mathcal{H})$ of all linear maps of $\mathcal{H}$ into itself. A subspace $\mathcal{K}$ of $\mathcal{H}$ is said to be $(\pi)$-invariant, if every representing operator $\pi(\mathscr{A})$ maps $\mathcal{K}$ into itself. A representation is called irreducible if it has some non-zero representing operators, and $\mathcal{H}$ is its only non-zero $(\pi)$-invariant subspace. Recall from [6] that, an ideal $P$ of $\mathscr{A}$ is called primitive if it is the kernel of some irreducible representation of $\mathscr{A}$, or equivalently, $P=(M: \mathscr{A})=\{a \in A \mid a \mathscr{A} \subseteq M\}$, for some maximal left ideal $M$ of $\mathscr{A}$.

Theorem 9. Let $\mathscr{A}$ be an algebra with unity and $n \in \mathbb{N}$. Then every primitive ideal of $\boldsymbol{T}_{n}(\mathscr{A})$ is of the form $P_{0} E_{k, k}+\sum_{k \neq i \leq j} \mathscr{A} E_{i, j}$, where $P_{0}$ is a primitive ideal of $\mathscr{A}$ and $1 \leq k \leq n$. Also, every primitive ideal of the rings $\boldsymbol{S}_{n}(\mathscr{A}), \boldsymbol{A}_{n}(\mathscr{A}), \boldsymbol{B}_{n}(\mathscr{A}), \boldsymbol{S}_{n}(\mathscr{A})$, and $\boldsymbol{V}_{n}(\mathscr{A})$ has a component $P_{0} I_{n}$, where $P_{0}$ is a primitive ideal of $\mathscr{A}$ (e.g., every primitive ideal of $\boldsymbol{S}_{n}(\mathscr{A})$ is of the form $\left.P_{0} I_{n}+\sum_{i<j} \mathscr{A} E_{i, j}\right)$.

Proof. Let $P$ be a primitive ideal of $\mathbf{T}_{n}(\mathscr{A})$. Then $P=\left(M: \mathbf{T}_{n}(\mathscr{A})\right)$ for some maximal left ideal $M$ of $\mathbf{T}_{n}(\mathscr{A})$. Let $N$ be the set of all elements of $\mathbf{T}_{n}(\mathscr{A})$ with zero main diagonal. Clearly, $N$ is a nilpotent ideal of $\mathbf{T}_{n}(\mathscr{A})$ and so $N \subseteq J\left(\mathbf{T}_{n}(\mathscr{A})\right) \subseteq M$, where $J\left(\mathbf{T}_{n}(\mathscr{A})\right)$ is the Jacobson radical of $\mathbf{T}_{n}(\mathscr{A})$. Also one can see that

$$
\varphi: \mathbf{T}_{n}(\mathscr{A}) / N \rightarrow \mathscr{A} \oplus \mathscr{A} \oplus \cdots \oplus \mathscr{A} \text {, given by } \varphi\left(\left(a_{i, j}\right)+N\right)=\left(a_{1,1}, a_{2,2}, \ldots, a_{n, n}\right),
$$

is an isomorphism. Since $M$ is a maximal left ideal of $\mathbf{T}_{n}(\mathscr{A}), M / N$ is a maximal left ideal of $\mathbf{T}_{n}(\mathscr{A}) / N$, then $\varphi(M / N)$ is a maximal left ideal of $\mathscr{A} \oplus \mathscr{A} \oplus \cdots \oplus \mathscr{A}$. Therefore, $M=M_{0} E_{k, k}+\sum_{k \neq i \leq j} \mathscr{A} E_{i, j}$, for some $1 \leq k \leq n$ and some maximal left ideal $M_{0}$ of $\mathscr{A}$. So $P=\left(M: \mathbf{T}_{n}(\mathscr{A})\right)=\left(M_{0}: \mathscr{A}\right) E_{k, k}+\sum_{k \neq i \leq j} \mathscr{A} E_{i, j}$. Thus $P_{0}=\left(M_{0}: \mathscr{A}\right)$ is a primitive ideal of $A$ and $P=P_{0} E_{k, k}+\sum_{k \neq i \leq j} \mathscr{A} E_{i, j}$. The other cases can be shown similarly. 
Let $\mathscr{A}$ be a commutative unital $\mathrm{C}^{*}$-algebra and $I$ be an ideal of $\mathscr{A}$. Then by $[5$, Theorem 5.4.4], $I$ is primitive if and only if it is maximal. As a consequence of this result, we obtain the following.

Corollary 10. Let $\mathscr{A}$ be a commutative unital $C^{*}$-algebra and let $n \in \mathbb{N}$. Then the primitive ideals of the Banach *-algebra $\boldsymbol{T}_{n}(\mathscr{A})\left(\right.$ resp., $\boldsymbol{S}_{n}(\mathscr{A}), \boldsymbol{A}_{n}(\mathscr{A}), \boldsymbol{B}_{n}(\mathscr{A}), \boldsymbol{U}_{n}(\mathscr{A})$, or $\boldsymbol{V}_{n}(\mathscr{A})$ ) are all maximal.

Let $X$ be a topological space and let $x$ and $y$ be points in $X$. Then $x$ and $y$ can be separated if each lies in a neighborhood which does not contain the other point. $X$ is a $\mathbf{T}_{1}$-space if any two distinct points in $X$ are separated, or equivalently, every singleton set of $X$ is closed.

The hull-kernel topology on the space of primitive ideals of a noncommutative algebra was first systematically studied by Nathan Jacobson [3]. Let $\mathscr{A}$ be an algebra with unity and $I$ be a subset of $\mathscr{A}$. Let $\operatorname{hull}(I)$ denote the set of primitive ideals of $\mathscr{A}$ containing $I$. If $J$ is a non-empty set of primitive ideals of $\mathscr{A}$, we denote by $\operatorname{ker}(J)$ the intersection of the ideals in $J$. We set $\operatorname{ker}(\emptyset)=\mathscr{A}$. The closure of a set $J$ of primitive ideals is $\bar{J}=h u l l(k e r(J))$. It is well-down that the topological space of primitive ideals of $\mathscr{A}$ is $\mathbf{T}_{1}$-space if and only if all primitive ideals of $\mathscr{A}$ are maximal.

Corollary 11. Let $\mathscr{A}$ be a unital commutative $C^{*}$-algebra. Then the primitive ideal space of the Banach *-algebra $\boldsymbol{T}_{n}(\mathscr{A})\left(\right.$ resp., $\boldsymbol{S}_{n}(\mathscr{A}), \boldsymbol{A}_{n}(\mathscr{A}), \boldsymbol{B}_{n}(\mathscr{A}), \boldsymbol{U}_{n}(\mathscr{A})$, or $\left.\boldsymbol{V}_{n}(\mathscr{A})\right)$ is a $\boldsymbol{T}_{1}$-space.

Acknowledgment. This research was done when the first author was a $\mathrm{PhD}$ student at Tarbiat Modares University. The research of the first author was partially supported by a grant (No. 96000090) from Iran National Science Foundation (INSF).

\section{References}

[1] O.M. Di Vincenzo, P. Koshlukov and R. La Scala, Involutions for upper triangular matrix algebras, Adv. in Appl. Math. 37, 541-568, 2006.

[2] J. Dixmier, $C^{*}$-Algebras, North-Holland, Amsterdam, 1977.

[3] N. Jacobson, A topology for the set of primitive ideals in an arbitrary ring, Proc, Nat, Acad, Sci. U.S.A. 31, 333-338, 1945.

[4] T.K. Lee and Y. Zhou, Armendariz and reduced rings, Comm. Algebra 32 (6), 22872299, 2004.

[5] G.J. Murphy, $C^{*}$-Algebras and Operator Theory Academic Press, 1990.

[6] T.W. Palmer, Banach Algebras and the General Theory of *-Algebras Volume I Algebras and Banach Algebras, Encyclopedia of Mathematics and its Applications, Vol. $1,1994$.

[7] V. Paulsen, Completely Bounded Maps and Operator Algebras, vol. 78, Cambridge University Press, 2002. 